\title{
Efficient Continuous Beam Steering for Planar Arrays of Differential Microphones
}

\author{
Alberto Bernardini, Student Member, IEEE, Matteo D'Aria, Roberto Sannino, \\ Augusto Sarti, Senior Member, IEEE
}

\begin{abstract}
Performing continuous beam steering, from planar arrays of high-order differential microphones, is not trivial. The main problem is that shape-preserving beams can be steered only in a finite set of privileged directions, which depend on the position and the number of physical microphones. In this letter, we propose a simple and computationally inexpensive method for alleviating this problem using planar microphone arrays. Given two identical reference beams pointing in two different directions, we show how to build a beam of nearly constant shape, which can be continuously steered between such two directions. The proposed method, unlike the diffused steering approaches based on linear combinations of eigenbeams (spherical harmonics), is applicable to planar arrays also if we deal with beams characterized by high-order polar patterns. Using the coefficients of the Fourier series of the polar patterns, we also show how to find a trade-off between shape invariance of the steered beam, and maximum angular displacement between the two reference beams. We show the effectiveness of the proposed method through the analysis of models based on first, second and third-order differential microphones.
\end{abstract}

\section{INTRODUCTION}

$\mathbf{T}$ $\mathrm{HE}$ interest in signal processing techniques for deriving directional Beamformers (BFs) from small-size arrays of omnidirectional microphones (e.g. arrays of MEMS microphones [1]) is steadily growing due to the proliferation of embedded systems for home automation, audio surveillance, infotainment, automotive engineering [2] and handsfree interaction [3]. Also emerging applications relying on arrays of compact sub-arrays (plenacoustic cameras [4], [5]) could benefit from such methodologies. As discussed in [6], there are two broad classes of beamforming methods applied to small-size microphone arrays, one based on additive [7][14] operations; and one based on differential [6], [15]-[22] operations on microphone signals. Worth mentioning are also hybrid techniques such as differential-integral approaches [23]. Differential Microphone Arrays (DMAs) require arrays of small size, as the distance between sensors must be small enough to satisfy the assumption that acoustic pressure field differentials are well approximated by differences between microphone signals [19]. The smaller the distances between sensors the wider the spectrum ranges in which the resulting

Copyright (c) 2017 IEEE. Personal use of this material is permitted. However, permission to use this material for any other purposes must be obtained from the IEEE by sending a request to pubs-permissions@ieee.org. A. Bernardini and A. Sarti are with the Dipartimento di Elettronica, Informazione e Bioingegneria, Politecnico di Milano, Piazza L. Da Vinci 32, 20133 Milano, Italy (e-mail: [alberto.bernardini,augusto.sarti]@polimi.it). M. D'Aria and R. Sannino are with STMicroelectronics s.r.l., Via Olivetti 2, 20864 Agrate Brianza (MB), Italy (e-mail: [matteo.daria,roberto.sannino]@st.com). beams are nearly frequency-invariant [6]. Moreover, the polar pattern of the beams can be easily shaped by adjusting the delays between the combined microphone signals. The literature is rich with DMA approaches employing different array geometries, such as: Uniform Linear Arrays (ULAs) [6], [18], [24]; NonUniform Linear Arrays (NULAs) [21]; and Uniform Circular Arrays (UCAs) [20]. The design of DMAs is characterized by rigid symmetry constraints. For example, using ULAs and NULAs we can only obtain beams that are symmetric with respect to the line on which the physical microphones are placed. This means that, if we want only one mainlobe in the directivity pattern, it must be aligned with one of the two possible directions along the above-mentioned line [6], [21]. Similarly, in the case of UCAs the symmetry axes of the resulting polar patterns can only be the lines joining the array center and the physical microphones on the circumference [20]. As explained in [20], applying alternative techniques to UCAs, such as superdirective beamforming without symmetry constraints, we can design directional microphones focusing to arbitrary directions, but the shape of the resulting polar pattern is strongly direction-dependent and symmetry can no longer be guaranteed.

Consequently, the development of beamforming methods for designing shape-preserving beams with high-order polar patterns, continuously steerable to arbitrary directions, is not straightforward. In this regard, Elko et al. [15], [25], [26], [27] developed a method, which is based on a linear combination of eigenbeams shaped like spherical harmonics of different orders, similarly to what done in (B-format) ambisonics coding [28], [29]. The method described in [25] uses differential configurations for building the needed eigenbeams [25]. How microphone gain and phase mismatches impact on the mainlobe misorientation, using such an approach [25], is also discussed in the literature [30], [31]. Disposing of 3D arrays (such as spherical arrays) composed of closely spaced microphones, eigenbeams of any order can in principle be built [27], enabling the construction of beams with arbitrary order and mainlobe orientation. However, the use of 3D array geometries is impractical in many embedded applications and 2D configurations would be preferred [32]. Nevertheless, if we are constrained to use planar (2D) arrays, the approach adopted by Elko et al. [27] works only for steering beams with first-order polar patterns, as higher-order spherical harmonics have complex 3D shapes [28], [29] and 3D array geometries become necessary [27].

In this letter, we propose a beamforming method applicable to $2 \mathrm{D}$ small-size array configurations for deriving a 
TABLE I

$N$-TH ORder BeAm PATtern COEFFICIENTS

\begin{tabular}{|c|c|c|c|c|c|c|}
\hline$N$ & $c_{0}$ & $c_{1}$ & $c_{2}$ & $c_{3}$ & $c_{4}$ & $c_{5}$ \\
\hline $\mathbf{1}$ & $1-a_{1}$ & $a_{1}$ & - & - & - & - \\
\hline $\mathbf{2}$ & $1-a_{1}-a_{2} / 2$ & $a_{1}$ & $a_{2} / 2$ & - & - & - \\
\hline $\mathbf{3}$ & $\begin{array}{c}1-a_{1}- \\
a_{2} / 2-a_{3}\end{array}$ & $\begin{array}{c}a_{1}+ \\
3 a_{3} / 4\end{array}$ & $a_{2} / 2$ & $a_{3} / 4$ & - & - \\
\hline $\mathbf{4}$ & $\begin{array}{c}1-a_{1}-a_{2} / 2- \\
a_{3}-5 a_{4} / 8\end{array}$ & $\begin{array}{c}a_{1}+ \\
3 a_{3} / 4\end{array}$ & $\begin{array}{c}a_{2} / 2+ \\
a_{4} / 2\end{array}$ & $a_{3} / 4$ & $a_{4} / 8$ & - \\
\hline $\mathbf{5}$ & $\begin{array}{c}1-a_{1}- \\
a_{2} / 2-a_{3}- \\
5 a_{4} / 8-a_{5}\end{array}$ & $\begin{array}{c}a_{1}+ \\
3 a_{3} / 4+ \\
5 a_{5} / 8\end{array}$ & $\begin{array}{c}a_{2} / 2+ \\
a_{4} / 2\end{array}$ & $\begin{array}{c}a_{1}+ \\
3 a_{3} / 4+ \\
5 a_{5} / 16\end{array}$ & $a_{4} / 8$ & $a_{5} / 16$ \\
\hline
\end{tabular}

continuously steerable beam starting from a pair of arbitraryorder reference beams, which are coincident in space and only differ from each other by a rotation. We show how and under which conditions such beams can be combined in order to obtain a steerable beam of matching shape using a simple weighted combination of patterns. We also propose a metric for assessing shape similarity between the resulting beams and offer an evaluation of the performance of the method.

\section{High-ORDER BEAM PATTERNS}

We will refer to a Beamformer (BF) as a combination of filtered versions of the signals coming from a microphone arrangement, designed to obtain a directional beam. It is known that a $\mathrm{BF}$ can be structured in a layered fashion; i.e. combining the output signals of other BFs. In fact, high-order DMAs are examples of BFs of the sort. A BF is always characterized by a spatial position and a beam pattern. The former corresponds to the fixed reference point of the microphone array. The latter can be defined as a pattern of order $N$ and, assuming that it does not depend on the frequency of the audio signal [19], expressed as:

$$
B(\theta)=a_{0}+\sum_{n=1}^{N} a_{n} \cos ^{n}(\theta) \text {, with } a_{0}=1-\sum_{n=1}^{N} a_{n},
$$

where $\theta$ is the azimuth of the pattern $(0<\theta \leq 2 \pi)$, and $a_{0}, \ldots, a_{N}$ are positive real coefficients in the range $[0,1]$. We also define the derivative of (1) as

$$
D_{B}(\theta)=\frac{\partial B(\theta)}{\partial \theta}=-\sum_{n=1}^{N} n a_{n} \sin (\theta) \cos ^{n-1}(\theta),
$$

which will be used later on. Notice that we can always express (1) in the form [33]

$$
B(\theta)=c_{0}+\sum_{n=1}^{N} c_{n} \cos (n \theta),
$$

where $c_{0}, \ldots, c_{N}$ are real coefficients, that can be computed as shown in Appendix A Such coefficients are collected in Table I up to order 5.

Later on, a generic beam pattern will be thought of as a signal for the purpose of introducing a metric for shape comparison. The "distance" between two pattern shapes, in fact, will be based on the "power" of the difference between the signals that describe such patterns. This distance, in turn, can be easily expressed as a function of the Fourier coefficients of the (periodic) signals that describe the patterns, thanks to Parseval's theorem. Starting from (3), in fact, it is quite straightforward to compute the coefficients $d_{n}$ of the Fourier series of $B(\theta)$ (which is a periodic function of the angle) as $d_{0}=c_{0}, d_{n}=c_{n} / 2$ for $0<|n| \leq N$ and $d_{n}=0$ for $|n|>N$. These coefficients are strictly related to the Fourier descriptors [34] that are used for describing the shape of closed curves. Let us now treat $B(\theta)$ like a signal, so that, using Parseval's theorem, we can readily compute the "power" (integral of the square modulus) of (3) as

$$
\mathbf{W}_{\mathbf{B}}=\sum_{n=-N}^{N}\left|d_{n}\right|^{2}=\left|c_{0}\right|^{2}+\frac{1}{2} \sum_{n=1}^{N}\left|c_{n}\right|^{2} .
$$

An alternate representation (in the frequency domain) of the beam pattern of a BF can be derived by computing the continuous Fourier transform of $B(\theta)$ (ignoring its periodicity). As a result, we obtain a "line spectrum" of the form

$$
\mathbf{B}(\psi)=d_{0} \delta(\psi)+\sum_{n=1}^{N} d_{n}\left(\delta\left(\psi-\frac{n}{2 \pi}\right)+\delta\left(\psi+\frac{n}{2 \pi}\right)\right)
$$

where $\psi$ is the domain of the Fourier transform, expressed in $\operatorname{rad}^{-1}, \delta($.$) is the Dirac distribution and the coefficients d_{n}$ are the amplitudes of the spectral lines.

Rotating $B(\theta)$ of the angle $\rho$, returns the beam pattern $\bar{B}(\theta)=B(\theta-\rho)$, which can be written as

$$
B(\theta-\rho)=c_{0}+\sum_{n=1}^{N} c_{n}(\cos (n \theta) \cos (n \rho)+\sin (n \theta) \sin (n \rho)) \text {. }
$$

The corresponding "line spectrum" will therefore be

$$
\begin{aligned}
& \overline{\mathbf{B}}(\psi)=\sum_{n=1}^{N}\left(d_{n} \cos (n \rho)\left(\delta\left(\psi+\frac{n}{2 \pi}\right)+\delta\left(\psi-\frac{n}{2 \pi}\right)\right)+\right. \\
& \left.+j d_{n} \sin (n \rho)\left(\delta\left(\psi+\frac{n}{2 \pi}\right)-\delta\left(\psi-\frac{n}{2 \pi}\right)\right)\right)+d_{0} \delta(\psi) .
\end{aligned}
$$

\section{Continuous Steering of Planar Patterns}

\section{A. Computing the weights}

Let us consider two identical beam patterns $B(\theta)$ and $\bar{B}(\theta)$, sharing the same location in space and pointing in two different directions $\theta_{0}$ and $\bar{\theta}$ with an angular displacement $\rho=\bar{\theta}-\theta_{0}<\pi / 2$. For simplicity, we assume $\theta_{0}=0$ and $\bar{\theta}=\rho$. We also assume that the two beam patterns $B(\theta)$ and $\bar{B}(\theta)$ are symmetric with respect to their pointing direction. We consider the weighted sum $B_{\text {sum }}(\theta)=\alpha B(\theta)+\bar{\alpha} \bar{B}(\theta)$, where $\alpha>0$ and $\bar{\alpha}>0$. This can be rewritten as

$$
B_{\text {sum }}(\theta)=\bar{\alpha}[\beta B(\theta)+B(\theta-\rho)]
$$

where $\beta=\alpha / \bar{\alpha}$. We now want to point the mainlobe of $B_{\text {sum }}(\theta)$ in a desired direction $\theta_{d}, 0<\theta_{d}<\rho$. This we do by ensuring that the derivative $D_{B_{\text {sum }}}(\theta)$ of (7) is zero at $\theta_{d}$. As $D_{B_{\text {sum }}}(\theta)=\bar{\alpha}\left[\beta D_{B}(\theta)+D_{B}(\theta-\rho)\right]$, we derive the constraint

$$
\beta=\frac{D_{B}\left(\theta_{d}-\rho\right)}{-D_{B}\left(\theta_{d}\right)}=\frac{\sin \left(\rho-\theta_{d}\right)}{\sin \left(\theta_{d}\right)} \frac{\sum_{n=1}^{N} n a_{n} \cos ^{n-1}\left(\theta_{d}-\rho\right)}{\sum_{n=1}^{N} n a_{n} \cos ^{n-1}\left(\theta_{d}\right)} .
$$


Notice that if $\theta_{d}=\rho / 2$, then $\beta=1$. In order to normalize $B_{\text {sum }}(\theta)$, we set $B_{\text {sum }}\left(\theta_{d}\right)=1$, which is guaranteed by

$$
\bar{\alpha}=\frac{1}{\beta B\left(\theta_{d}\right)+B\left(\theta_{d}-\rho\right)},
$$

from which we can compute $\alpha$ as $\alpha=\beta / \bar{\alpha}$. $B_{\text {sum }}(\theta)$ is the beam pattern of a directional $\mathrm{BF}$ whose main lobe has a maximum in $\theta_{d}$. Fig. 1. Fig. 2, Fig. 3 and Fig. 4 show some beam patterns derived using the presented approach.
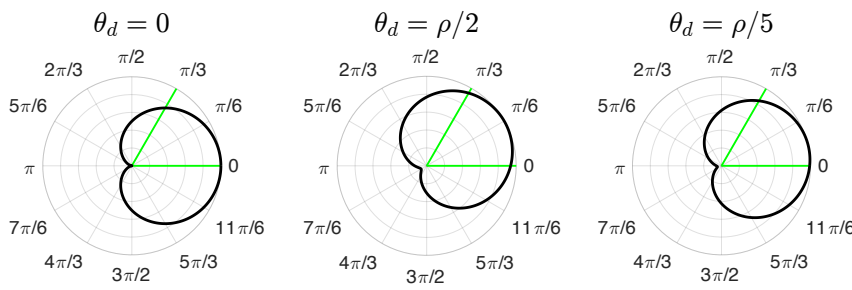

Fig. 1. A first-order (reference) pattern $B(\theta)$ with $a_{1}=0.5$ (left); and two patterns derived from $B(\theta)$ and $B(\theta-\rho)$ with $\rho=\pi / 3$ (center and right) The directions of the reference patterns are marked with green radial lines.
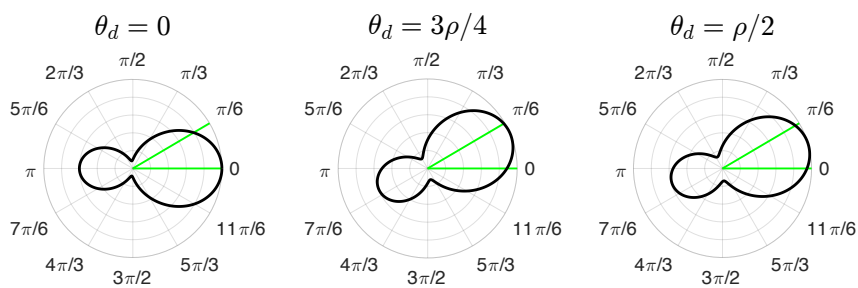

Fig. 2. A second-order (reference) pattern $B(\theta)$ with $a_{1}=0.2$ and $a_{2}=0.7$ (left); and two patterns derived from $B(\theta)$ and $B(\theta-\rho)$ with $\rho=\pi / 5.9$ (center and right).
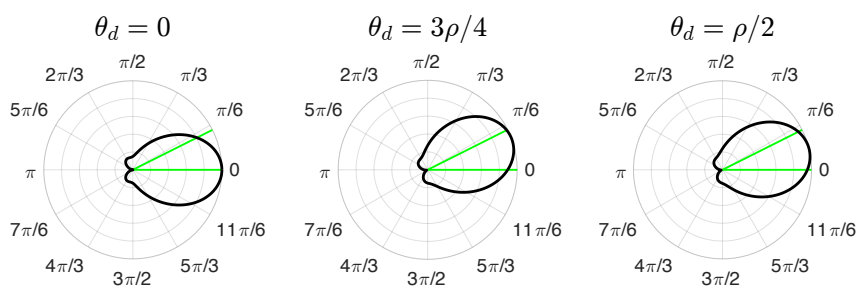

Fig. 3. A third-order (reference) pattern $B(\theta)$ with $a_{1}=0.1, a_{2}=0.35$ and $a_{3}=0.4$ (left); and two patterns derived from $B(\theta)$ and $B(\theta-\rho)$ with $\rho=\pi / 6.758$ (center and right).
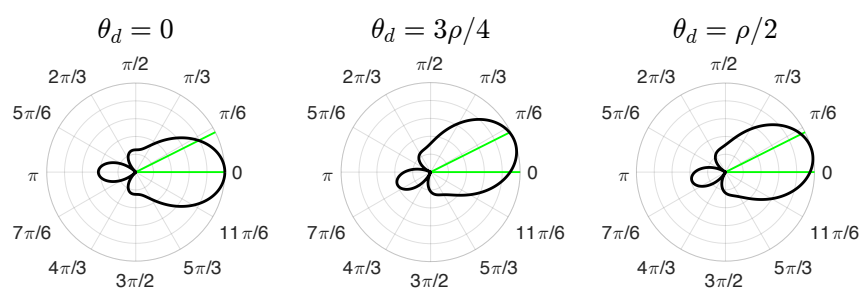

Fig. 4. A third-order (reference) pattern $B(\theta)$ with $a_{1}=0.01, a_{2}=0.04$ and $a_{3}=0.7$ (left); and two patterns derived from $B(\theta)$ and $B(\theta-\rho)$ with $\rho=\pi / 6.758$ (center and right).

\section{B. Beam Similarity Error}

Though similar, $B_{\text {sum }}(\theta)$ is generally different from the reference pattern $B\left(\theta-\theta_{d}\right)$. We would like, however, to exercise some control over the "degree of similarity" of such beam patterns through a careful selection of the parameters $a_{n}, \rho$ and $\theta_{d}$. In order to do so, we define the shape error

$$
E(\theta)=B_{\text {sum }}(\theta)-B\left(\theta-\theta_{d}\right) .
$$

and, using the definitions (5), (6) and (4), we derive the power of its spectrum $\mathbf{E}(\psi)$

$$
\begin{aligned}
& \mathbf{W}_{\mathbf{E}}=\frac{1}{2} \sum_{n=1}^{N}\left(\left|c_{n}\left(\bar{\alpha} \beta+\bar{\alpha} \cos (n \rho)-\cos \left(n \theta_{d}\right)\right)\right|^{2}+\right. \\
& \left.+\left|c_{n}\left(\bar{\alpha} \sin (n \rho)-\sin \left(n \theta_{d}\right)\right)\right|^{2}\right)+\left|c_{0}(\bar{\alpha} \beta+\bar{\alpha}-1)\right|^{2} .
\end{aligned}
$$

We then define the percentage similarity error

$$
J_{E}=100 \times \frac{\mathbf{W}_{\mathbf{E}}}{\mathbf{W}_{\mathbf{B}}},
$$

which is expected to be small when $B_{\text {sum }}(\theta)$ and $B\left(\theta-\theta_{d}\right)$ are similar. Given $\rho$ and $a_{i}$, the largest $J_{E}$ always corresponds to $\theta_{d}=\rho / 2$, which is the only one angle in which the beam pattern $B_{\text {sum }}(\theta)$ is fully symmetric with respect to its main lobe direction (like $B(\theta)$ ). These facts can be readily verified in Fig. 1. where the largest value $J_{E} \simeq 0.512$ is reached at $\theta_{d}=\rho / 2$, where at $\theta_{d}=\rho / 5$ we have $J_{E} \simeq 0.219$.

In Fig. 5] we show the values of $J_{E}$ as a function of a generic first-order beam pattern, assuming all the possible values of $a_{1}$ in the range $[0,1]$ and $\rho$ in the range $[0, \pi / 3]$. The maximum of the function in this plot is $J_{E}^{*} \simeq 0.546$. Let us assume in a

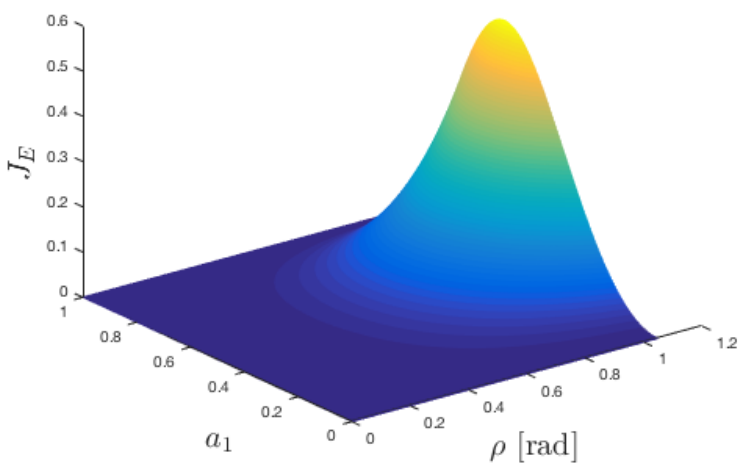

Fig. 5. Percentage similarity error $J_{E}$ as a function of $\rho$ and $a_{1}$. The maximum of $J_{E}$ is $J_{E}^{*} \simeq 0.546$ with $a_{1}=0.569$.

reference application $J_{E}^{*}$ be the maximum allowed percentage similarity error; we want to find the corresponding maximum allowed $\rho$ for each beam pattern order $N$, assuming the parameters $a_{n}$ can vary arbitrarily. Fig. 6 shows the maximum allowed $\rho$ for the first 5 orders, fixing $\theta_{d}=\rho / 2$ and picking the "worst" combination of $a_{n}$ parameters. The values of $\rho$ plotted in Fig. 6 for the first 3 orders are used also in Fig. 1 , Fig. 2 Fig. 3 and Fig. 4

\section{Approximate computation of $\beta$}

In real-time applications in which $\theta_{d}$ is time-varying and a high update rate for the non-uniform weights is required, 


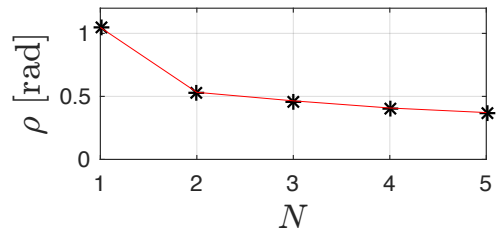

Fig. 6. Maximum allowed $\rho$ for the first 5 orders, corresponding to a maximum allowed percentage similarity error of $J_{E}^{*} \simeq 0.546$.

it might be useful to consider simpler expressions, which approximate (9) and (8), for realizing even cheaper beam steering implementations. While 9 is difficult to simplify using a general form, in many cases some properties of (8) can be exploited. Firstly, we notice that, in the first-order case, (8) becomes $\hat{\beta}=\sin \left(\rho-\theta_{d}\right) / \sin \left(\theta_{d}\right)$. Actually, $\hat{\beta}$ is a good simplification also for higher order beam patterns as the ratio of summations in $(8)$ is often close to 1 . As for small angles $y$ the approximation $\sin (y) \approx y$ is reasonable, a further rougher simplification is $\tilde{\beta}=\left(\rho-\theta_{d}\right) / \theta_{d}$. It is worth saying that using $\hat{\beta}$ or $\tilde{\beta}$ some accuracy is lost in terms of mainlobe orientation, as $B\left(\theta_{d}\right)$ may not be anymore the maximum of $B(\theta)$. However, we have experimentally verified that the use of $\hat{\beta}$ or $\tilde{\beta}$ may lead to BFs with lower $J_{E}$. Therefore, $\hat{\beta}$ or $\tilde{\beta}$ could be fruitfully used in applications in which high orientation accuracy is not required.

\section{FREQUENCY-DEPENDENT BEAM PATTERNS}

The beam steering approach described in Section III relies on frequency-independent beam patterns, therefore we expect it to be well-suited for DMAs, which are known to exhibit a near-constant behavior over a wide range of frequencies [6]. In order to show that, we now apply the weight-derivation procedure described in Section III to the mildly frequencydependent beam patterns that we encounter in the case of linear DMAs and we show that the resulting weights can be well approximated, over a wide range of frequencies, by frequencyindependent weights.

As a relevant example, let us consider a compact arrangement of $M$ omnidirectional microphones made of a pair of coincident small-size ULAs, and let $\rho$ be the angle between them. We can express a frequency-dependent beam pattern obtained from a differential ULA as

$$
B(\theta, \omega)=\sum_{m=1}^{M} H_{m}(\omega) e^{j(m-1) \omega \tau_{0} \cos \theta},
$$

where the filters $H_{m}(\omega)$ are designed as described in [35]; $\omega=2 \pi f$ is the normalized temporal frequency $(f>0$ is the temporal frequency in hertz); $\tau_{0}=\delta / c$ is the delay between two adjacent microphones at the angle $\theta=0 ; \delta$ is the spacing between each pair of microphones; and $c$ is the speed of sound. From this expression we can readily compute the derivative $D(\theta, \omega)$ of $B(\theta, \omega)$ with respect to the angle $\theta$ as well as a general expression $B(\theta-\rho, \omega)$ for the rotated version of $B(\theta, \omega)$. Finally, we can compute the frequencydependent weights $\alpha(\omega)$ and $\bar{\alpha}(\omega)$ as described in the previous Sections. Fig. 7 shows a comparison between the weights that are computed using frequency-dependent and frequencyindependent beam patterns of cardioids up to order three. As
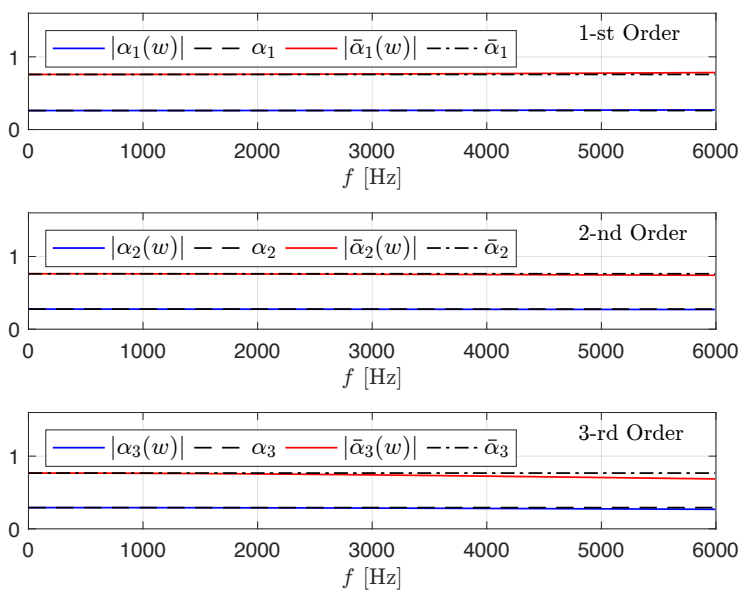

Fig. 7. Comparison between frequency-independent weights and frequencydependent weights. The subscripts in the legends indicate the order of the reference patterns. The imaginary part of frequency-dependent weights is very small with respect to the real part. The reference beam pattern is the $N$-th order cardioid $B(\theta)=(0.5+0.5 \cos (\theta))^{N}$. The frequency-dependent beam pattern is computed using eq. 13 We set $M=2$ for $N=1, M=3$ for $N=2$ and $M=4$ for $N=3$. The other parameters are $\rho=\pi / 5$, $\theta_{d}=3 \rho / 4, \delta=0.0075 \mathrm{~m}$ and $c=340 \mathrm{~m} / \mathrm{s}$.

we can see, the deviations are always minimal (visible only for higher-order DMAs and at higher frequencies).

As expected, we also verified that the frequency dependence is more pronounced for larger values of $\delta$.

\section{COnClusions}

In this letter we showed that, starting from two identical (reference) beams pointing in different directions, it is possible to construct a closely matching beam pointing anywhere in between, as a linear combination of the reference beams. We show under which conditions this operation can be done in a shape-preserving fashion. In particular, using the Fourier descriptors of the reference polar patterns, we showed how to find a trade-off between the shape invariance of the steered beam, and the maximum angular displacement between the two reference beams. The method proved particularly promising for applications such as DMA-based beamforming.

\section{APPENDIX A \\ DERIVATION OF COEFFICIENTS $c_{n}$}

The coefficients $c_{n}$ in eq. (3) are derived using trigonometric power-reduction formulas [36] and rewriting (1) as

$$
B(\theta)=1-\sum_{n=1}^{N} a_{n}+\sum_{n=1}^{N} a_{n}\left(o_{n}+e_{n}\right)
$$

where $o_{n}$ is defined as

$$
o_{n}=\frac{(-1)^{n+1}+1}{2^{n}} \sum_{k=0}^{(n-1) / 2}\left(\begin{array}{l}
n \\
k
\end{array}\right) \cos ((n-2 k) \theta)
$$

and $e_{n}$ is defined as

$e_{n}=\frac{(-1)^{n}+1}{2^{n+1}}\left(\left(\begin{array}{c}n \\ \frac{n}{2}\end{array}\right)+2 \sum_{k=0}^{n / 2-1}\left(\begin{array}{l}n \\ k\end{array}\right) \cos ((n-2 k) \theta)\right)$. 


\section{REFERENCES}

[1] G. W. Elko, F. Pardo, D. Lopez, D. Bishop, and P. Gammel, "Surfacemicromachined mems microphone," in Proc. AES 115th Convention, vol. 1, Oct. 2003, pp. 1-8.

[2] M. Buck and M. Rodler, "First order differential microphone arrays for automotive applications," in Proc. IWAENC, vol. 1, Sep. 2001.

[3] G. W. Elko, "Microphone array systems for hands-free telecommunication," Speech Communications, Elsevier, vol. 20, no. 3-4, pp. 229-240, Dec. 1996.

[4] D. Markovic, F. Antonacci, A. Sarti, and S. Tubaro, "Soundfield imaging in the ray space," IEEE Transactions on Audio, Speech, and Language Processing, vol. 21, no. 12, pp. 2493-2505, Dec 2013.

[5] - "Multiview soundfield imaging in the projective ray space," IEEE/ACM Transactions on Audio, Speech, and Language Processing, vol. 23, no. 6, pp. 1054-1067, June 2015.

[6] J. Benesty and J. Chen, Study and Design of Differential Microphone Arrays, 1st ed., Springer, Ed., 2013.

[7] M. Branstein and D. B. Ward, Microphone Arrays: Signal Processing Techniques and Applications, 1st ed., Springer-Verlag, Ed., 2001.

[8] Y. Huang, J. Benesty, and J. Chen, Acoustic MIMO Signal Processing, 1st ed., Springer-Verlag, Ed., 2006.

[9] J. Benesty, J. Chen, and Y. Huang, Microphone Array Signal Processing, 1st ed., Springer-Verlag, Ed., 2008.

[10] O. L. Frost III, "An algorithm for linearly constrained adaptive array processing," Proc. IEEE, vol. 60, no. 8, pp. 926-935, Aug. 1972.

[11] W. F. Gabriel, "Adaptive arrays - an introduction," Proc. IEEE, vol. 64, no. 2, pp. 239-272, Feb. 1976.

[12] R. A. Monzigo and T. W. Miller, Introduction to Adaptive Arrays, 2nd ed., Wiley, Ed., 1980.

[13] T. J. Shan and T. Kailath, "Adaptive beamforming for coherence signals and interference," IEEE Transactions on Acoustics, Speech and Signal Processing, vol. ASSP-33, no. 3, pp. 527-536, June 1985.

[14] H. Cox, R. M. Zeskind, and M. M. Owen, "Robust adaptive beamforming," IEEE Transactions on Acoustics, Speech and Signal Processing, vol. ASSP-35, no. 10, pp. 1365-1376, Oct. 1987.

[15] G. W. Elko and A. T. N. Pong, "A steerable and variable first order differential microphone array," in Proc. IEEE International Conference on Acoustics, Speech and Signal Processing (ICASSP), vol. 1, Apr. 1997.

[16] H. Teutsch and G. W. Elko, "First- and second- order adaptive differential microphone arrays," in Proc. IWAENC, vol. 1, Sep. 2001.

[17] M. Buck, "Aspects of first-order differential microphone arrays in the presence of sensor imperfections," European Transactions on Telecommunications, vol. 13, no. 1, pp. 115-122, Mar.-Apr. 2002.

[18] G. W. Elko, "Differential microphone arrays," in Audio Signal Processing for Next-Generation Multimedia Communication Systems, Y. Huang and J. Benesty, M. K. Norwell, Ed., 2004.

[19] E. De Sena, H. Hacihabiboglu, and Z. Cvetkovic, "On the design and implementation of higher order differential microphones," IEEE Transactions on Audio, Speech, and Language Processing, vol. 20, no. 1, pp. 162-174, January 2012.

[20] J. Benesty, J. Chen, and I. Cohen, Design of Circular Differential Microphone Arrays, 1st ed., Springer, Ed., 2015.

[21] H. Zhang, J. Chen, and J. Benesty, "Study of nonuniform linear differential microphone arrays with the minimum-norm filter," Applied Acoustics, Elsevier, vol. 98, pp. 62-69, Nov 2015.

[22] J. Benesty, J. Chen, and C. Pan, Fundamentals of Differential Beamforming, 1st ed., Springer, Ed., 2016.

[23] T. D. Abhayapala and A. Gupta, "Higher order differential-integral microphone arrays," The Journal of the Acoustical Society of America, vol. 127, no. 1, pp. EL227-EL233, May 2010.

[24] C. Pan, J. Chen, and J. Benesty, "Theoretical analysis of differential microphone array beamforming and an improved solution," IEEE/ACM Transactions on Audio, Speech, and Language Processing, vol. 23, no. 11, pp. 2093-2105, Nov 2015.

[25] G. W. Elko, "Steerable and variable first-order differential microphone array," Patent US 006041127 A, Mar. 21, 2000.

[26] G. W. Elko, R. A. Kubli, and J. M. Meyer, "Audio system based on at least second-order eigenbeams," Patent WO 2003061336 A1, July 24, 2003.

[27] G. W. Elko and J. M. Meyer, "Polyhedral audio system based on at least second-order eigenbeams," Patent US9 197962 B2, Nov. 24, 2015.

[28] P. Felgett, "Ambisonics. part one: general system description," Studio Sound, pp. 20-22, 40, Aug. 1975.

[29] M. A. Gerzon, "Ambisonics. part two: studio techniques," Studio Sound, pp. 24-30, Oct. 1975
[30] X. Wu, H. Chen, J. Zhou, and T. Guo, "Study of the mainlobe misorientation of the first-order steerable differential array in the presence of microphone gain and phase errors," IEEE Signal Processing Letters, vol. 21, no. 6, pp. 667-671, June 2014.

[31] X. Wu and H. Chen, "Directivity factors of the first-order steerable differential array with microphone mismatches: Deterministic and worstcase analysis," IEEE/ACM Transactions on Audio, Speech, and Language Processing, vol. 24, no. 2, pp. 300-315, Feb 2016.

[32] H. Chen, T. D. Abhayapala, and W. Zhang, "Theory and design of compact hybrid microphone arrays on two-dimensional planes for threedimensional soundfield analysis," The Journal of the Acoustical Society of America, vol. 138, no. 5, pp. 3081-3092, 2015.

[33] L. Zhao, J. Benesty, and J. Chen, "Design of robust differential microphone arrays with the Jacobi - Anger expansion," Applied Acoustics, vol. 110 , pp. $194-206,2016$.

[34] C. T. Zahn and R. Z. Roskies, "Fourier descriptors for plane closed curves," IEEE Transactions on Computers, vol. C-21, no. 3, pp. 269281, March 1972.

[35] L. Zhao, J. Benesty, and J. Chen, "Design of robust differential microphone arrays," IEEE/ACM Transactions on Audio, Speech, and Language Processing, vol. 22, no. 10, pp. 1455-1466, Oct. 2014.

[36] W. H. Beyer, CRC Standard Mathematical Tables, 28th ed. Boca Raton, FL: CRC Press, 1987. 